\title{
An Adaptive Processing of Linear Array for Target Detection Improvement
}

\author{
V.Anitha ${ }^{1}$, S. Sri Jaya Lakshmi ${ }^{2}$, I.Sreedevi ${ }^{3}$, Habibulla Khan ${ }^{4}$, K. Sarat Kumar ${ }^{5}$ \\ P.Ramakrishna ${ }^{6}$
}

$1 \mathrm{M}$ Tech student, Department of ECE, $\mathrm{K} L$ University

2 Women scientist, Department of ECE, K L University

3, 6 B Tech students, Department of ECE, K L University

4, 5 Professors, Department of ECE, K L University

K L University, Vaddeswaram, Guntur-522502, India.

\begin{abstract}
Antenna arrays yield multiple, simultaneous available beams. These beams can be made to have high gain, low side lobes and controlled beam width. In conventional beam forming, the smallest beam width depends on the geometric dimensions of the receiving array. This problem may overcome by the Hyper beam invention. The linear array when implemented using Hyper beam technique, there is a considerable reduction of side lobes and beam width compared to the conventional beam forming. As a result, the relevant equations pertaining to normal linear array and the Hyper linear array are presented. The effect of the Hyper beam exponent on the beam patterns are shown.
\end{abstract}

\section{Keywords}

Hyper beam, Linear Array Antenna, side lobe level, beam width.

\section{INTRODUCTION}

Beam forming is the formation of beam in the desired direction only. There are some beam forming techniques to take the beam in the desired direction and suppress in other direction. Here we use hyper beam technique, to suppress the side lobes and grating lobes.

Beam forming techniques can be broadly classified into following categories:

$>$ Conventional beam formers

$>$ Adaptive beam formers

$>$ Desired signal maximization mode

$>$ Interference signal minimization or cancellation mode

Conventional beam formers use a fixed set of weightings and time-delays (or phasing) to combine the signals from the sensors in the array, primarily using only information about the location of the sensors in space and the wave directions of interest. In contrast, adaptive beam forming techniques generally combine this information with properties of the signals actually received by the array, typically to improve removal of unwanted signals from other directions, and get the data in only one particular direction. This process may be carried out in either the time or the frequency domain.

\section{METHODOLOGY}

Noise reduction and improvement of detecting the target is a successful design of a high performance system. While the classic way is to increase the array's size, constraints as integration, size and cost require new technical approaches like non-classical beam forming techniques. A new beam forming technique, called hyper beam is presented. As a result of the hyper beam offers high detection performance like beam width reduction, the target bearing estimation and reduces false alarm i.e., side lobe suppression.

\subsection{Hyper Beam}

The hyper beam forming by means of half beams, this is illustrated by a series of directivity patterns for a linear, planar and circular transducer. Moreover the influence of isotropic noise and non- isotropic noise sources as well as the separation of multiple targets are examined. The twodimensional Hyper beam focusing in one plane which contain the main beam direction, in order to achieve even greater reduction of beam width and the side lobes around the main beam in all directions.

The hyper beam technique yields a very narrow beam width with suppressed side lobe levels. The narrowness of beam width and side lobe suppression level depends on the variation of exponent value, which also leads to the suppression of grating lobe and also reduction of received noise level.

\subsection{Generation of Hyper Beam}

In principle of hyper beam generation a simple linear transducer used is shown in Fig. 1.The element spacing in one direction is half a wave length $(\lambda / 2)$ in order to allow beam steering in that particular direction without steering, i.e. all elements have the same phase or all elements are connected in parallel, conventionally beam forming is done by summing up all the transducer elements.

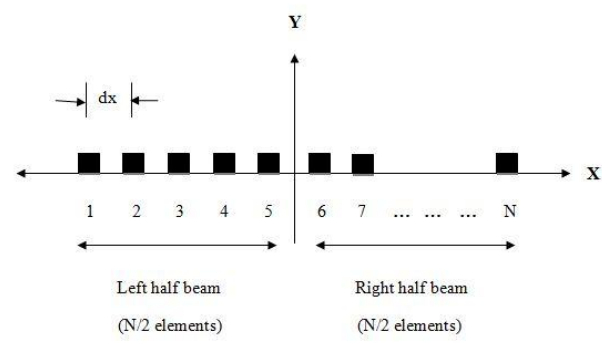

Figure 1 Simple linear transducer for Hyper beam generation 


\subsection{Forming of a hyper beam for linear array}

The forming of the hyper beam, which approaches to some extent the above mentioned ideal beam, shall now be illustrated by means of the beam pattern of Fig. 2 . applicable to the proposed linear transducer in the Fig. 1. First, the linear array is split into two equal half beams.

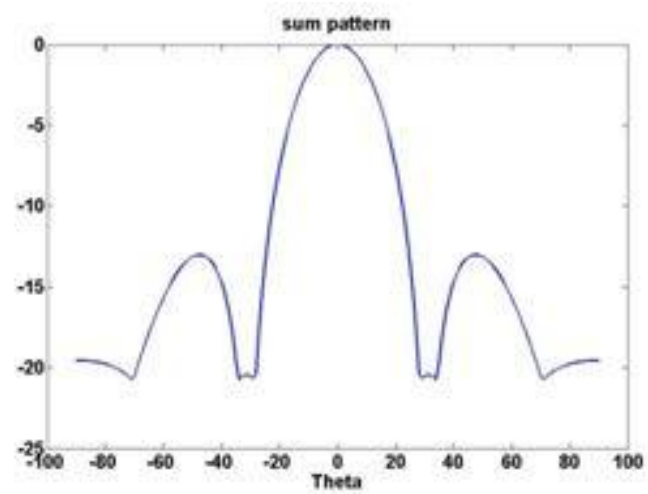

Figure 2 2D Sum pattern of 10 linear array

The sum beam can be created by complex summation of the left and right half beams. The beam magnitude, which is plotted in the beam patterns, is identical for the left and right half beams, and also identical to the sum of the magnitudes of both half beams because of the normalized plot as shown in Fig. 2 .

Difference beam is magnitude of the difference of the right half beam signal subtracted from the left half beam signal by considering the phases of the signals.

It is obvious that the values of the difference beam at each given direction are always lower or equal to those of the half beam. Furthermore the difference beam has a minimum in the direction of the sum beam at $0^{0}$ as shown in Fig. 3.

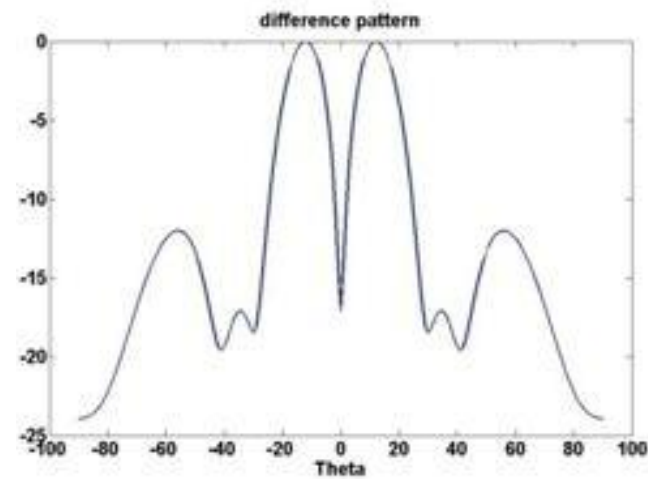

Figure 3 2D Difference pattern of 10 linear array Having recognized this interrelation, it is only a stone throw to the idea of subtracting the magnitude of the difference pattern from the half beam pattern. Of course the subtraction operation has to be performed rather on the magnitude numbers themselves, and not on the magnitude levels. The resulted simple hyper beam in 2D simulated for

10 linear array with exponent value $u=1$ is as shown in Fig. 4.

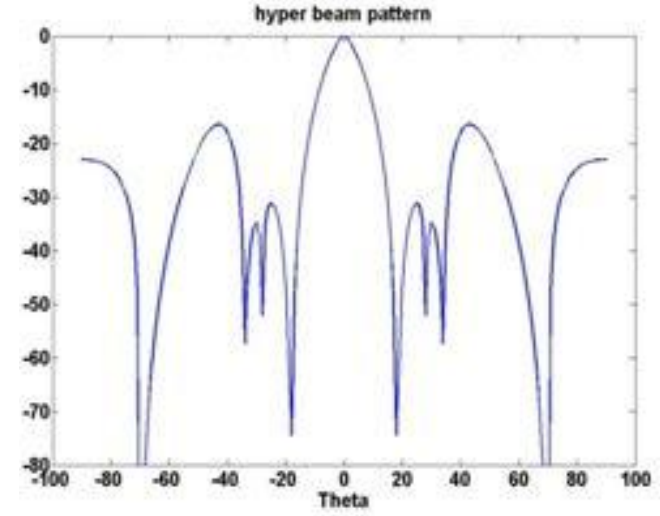

Figure 4 2D Simple Hyper Beam Pattern of 10 linear array with exponent value $u=1$

\section{MATHEMATICAL FORMULATIONS}

The equations for the creation of sum, difference and simple hyper beam are as follows:

The array factor equation for simple linear array is

$$
\begin{aligned}
& A F(\theta)=\sum_{n=1}^{N} I_{n} e^{j(n-1) k d[\sin \theta \cos \varphi+\alpha]}{ }_{n} \\
& \alpha_{n}=-\sin \theta_{0} \cos \varphi_{0}, \quad n=1,2, \ldots \ldots ., N
\end{aligned}
$$

The sum pattern is calculated from two half beams is given by

$$
\mathrm{S}(\theta)=\left|R_{L}\right|+\left|R_{R}\right|
$$

The difference pattern is calculated from below equation

$$
\mathrm{D}(\theta)=\left|R_{L}-R_{R}\right|
$$

Then the equation to obtain simple Hyper beam is

$$
R_{\text {hyp }}=\left|R_{L}\right|+\left|R_{R}\right|-\left|R_{L}-R_{R}\right|
$$

The equation of the general hyper beam is a function of the hyper beam exponent $u$ :

$$
R_{\text {hyp }}=\left\{\left(\left|R_{L}\right|+\left|R_{R}\right|\right)^{u}-\left(\left|R_{L}-R_{R}\right|\right)^{u}\right\}^{1 / u}
$$

Where

$$
\begin{aligned}
& \mathrm{R}_{\mathrm{L}}=\sum_{\mathrm{n}=1}^{\mathrm{N} / 2} \mathrm{e}^{\mathrm{j}(\mathrm{n}-1) \mathrm{dd}(\sin \theta \cos \varphi-\sin \theta \cos \varphi)} 0 \\
& 0 \\
& \mathrm{R}_{\mathrm{R}}=\sum_{\mathrm{n}=\mathrm{N} / 2}^{\mathrm{N}} \mathrm{e}^{\mathrm{j}(\mathrm{n}-1) \mathrm{dd}\left(\sin \theta \cos \varphi-\sin \theta_{0} \sin \varphi\right)} 0 \\
& 0 \\
& \text { where } \mathrm{k}=2 \pi / \lambda \\
& \text { And ' } \mathrm{u} \text { ' ranges from } 0.1 \text { to } 1 .
\end{aligned}
$$

\section{RESULTS}

With the Hyper beam effect, reduction of beam width and side lobes can be amplified and controlled. By varying the exponent value $\mathrm{u}$, different hyper beam patterns are obtained. As from the results the side lobe level and half power beam width is decreasing as the exponent value is decreased. For $\mathrm{u}=1$, the half power beam width for 10 element linear array is 8 degrees where as for $\mathrm{u}=0.1$ the half power beam width is reduced to 4 degrees as shown in Fig 6 . The resulted simple hyper beam in $2 \mathrm{D}$ simulated for a 10 linear array with exponent value $u=0.5$ is also shown 
in Fig. 5 where the half power beam width is observed as 6 degrees.

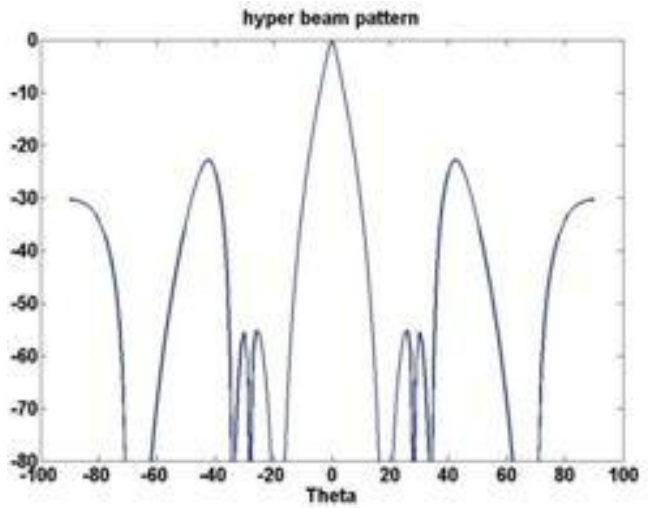

Figure 5 2D Hyper Beam Pattern of 10 linear array with exponent value $u=0.5$

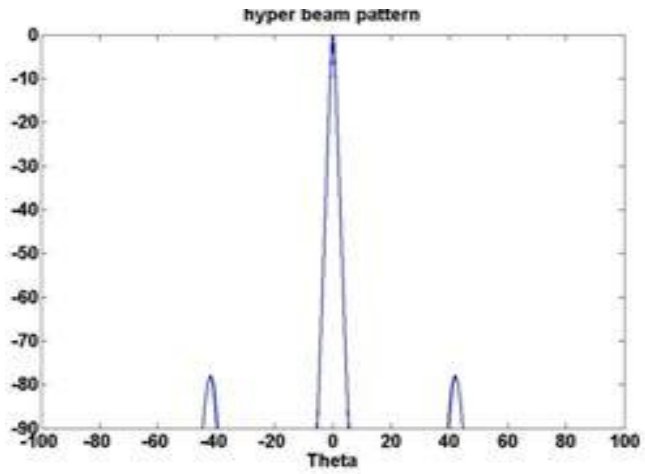

Figure 6 2D Hyper Beam Pattern of 10 linear array with exponent value $u=0.1$

From Fig 6 and Fig 7, for a 10 element linear array, conventional beam forming has the side lobe level and half power beam width $-13 \mathrm{~dB}$ and 14 degrees where as for Hyper beam forming technique, the side lobe level and the half power beam width is reduced to $-80 \mathrm{~dB}$ and 2 degrees respectively. Therefore, in comparison to conventional beam forming Hyper beam technique allows simultaneous reduction of beam width and the side lobes.

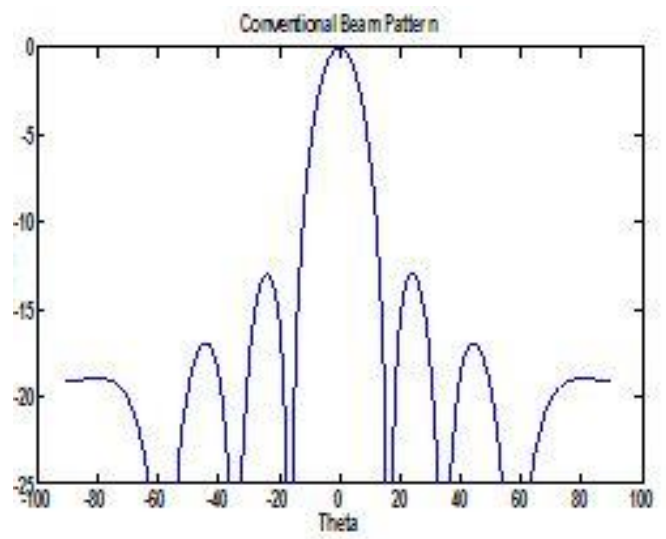

Figure 7 2D Conventional Beam Pattern of 10 linear array

To achieve point-to-point communication at higher frequencies, a single narrow beam of the radiation pattern is required which is usually obtained by multiunit linear arrays. Therefore, by varying number of elements in the array, the beam width and half power beam width is getting reduced as from the results. For a 10 linear array with exponent value $u=0.1$, the side lobe level is $-80 \mathrm{~dB}$ where as for a 15 element linear array with the same exponent value, the side lobe level is $-120 \mathrm{~dB}$ as shown in Fig 8 . The resulted simple hyper beam in $2 \mathrm{D}$ simulated for a 20 element linear array with exponent value $u=0.1$ is also shown in Fig. 9 where the side lobe level is observed as $-130 \mathrm{~dB}$. Similarly half power beam width is 2 degrees

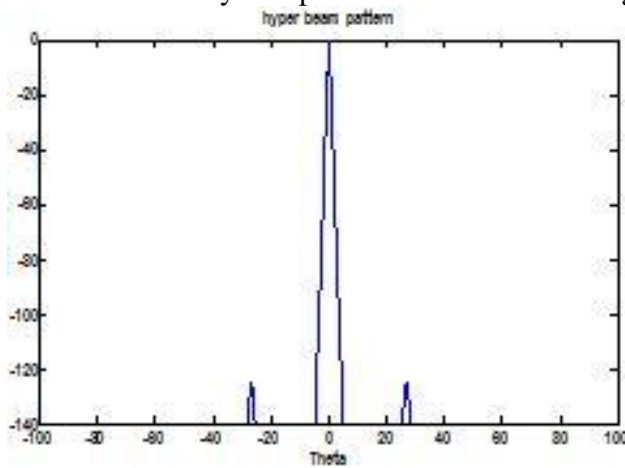

Figure 8 2D Hyper Beam Pattern of 15 linear array with exponent value $u=0.1$

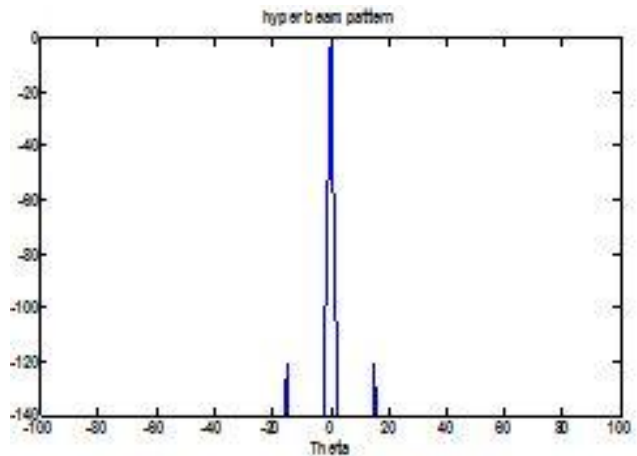

Figure 9 2D Hyper Beam Pattern of 20 linear array with exponent value $u=0.1$

\section{CONCLUSIONS}

The hyper beam technique yields a very narrow beam width with suppressed side lobe levels. The narrowness of beam width and side lobe suppression level depends on variation of exponent value, which also leads to the suppression of grating lobe for certain array configurations and also reduction of received noise level.

It has been proved that the Hyper beam technique is much more effective than the conventional beam forming where the high grade reception of data is allowed with an increased dynamic range and improved target bearing estimation. This is not only applicable for high frequency Surface wave Radar systems but also for the other communication systems.

\section{ACKNOWLEDGEMENTS}

The authors would like to thank the management of KL University, Vijayawada for excellent encouragement during the tenure of work. 


\section{REFERENCES}

[1] R.J.Mailloux, "Phased array architecture for millimetric active arrays", IEEE Antennas and Propagation Society Newsletter, Feb 1986.

[2] John D. Kraus, "Antennas", Mc GRAW -Hill, Newyork, 1950.

[3] Heiko Schliter, "Sonar Detection Improvement by Hyper Beam Technique".

[4] Heiko Schliter, "Method for the formation of radiated beams in direction finder systems".

[5] Constantine A. Balanis, "Antenna Theory Analysis and Design," John Wiley \& sons, Inc., USA 1982.

[6] R. S. Walker, "Bearing accuracy and resolution bounds of high-resolution beam formers," in Proc. IEEE ICASSP '85, Tampa.

[7] "Beam forming with aperture extrapolation (APEX) Performance in practice," in Proc. IEEE ICASSP '87, Dallas, 1987, Paper 47.9.

[8] J. K. Hsiao, "Normalized relationship among errors and side lobe levels," Radio Sci., vol. 19, pp. 292 302, Jan. 1984.

[9] S. P. Applebaum and D. J. Chapman, "Adaptive arrays with main beam constraints," ZEEE Trans. Antennas Propagat., vol. AI-24, pp. 650-662, Sept. 1976.

[10] K. Takao and K. Komiyama, "An adaptive antenna array under directional constraint," IEEE Trans. Antennas Propagat., vol. AI- 24, pp. 662-669, Sept. 1976.

[11] K. M. Buckley and L. J. Griffiths, "An adaptive generalized sidelobe canceller with derivative constraints," ZEEE Trans. Antennas Propagat., vol. AP-34, pp. 311-319, Mar. 1986.

[12] Arun K. Bhattacharayya, "Phased Array Antennas", Wiley series in Microwave and Optical Engineering, Chapter 5

[13] Yao Xu; Cao Xiang-yu; Yang Qun; Wang Pan-pan, "Pattern synthesis for adaptive antenna array with compensation of effects on mutual coupling".
[14] 14.David N.Swingler and Robert S.Walker, "LineArray Beamforming Using Linear Prediction for Aperture Interpolation and Extrapolation".

[15] Titus Lo and John Litva, "Adaptive Beam-Space Nulling of Multipath Signals".

[16] W. P. M. N. Keizer, "Low-sidelobe pattern synthesis using iterative Fourier techniques coded inMATLAB," IEEE Antennas Propag. Mag., vol. 51, no. 2, pp. 137-150, Apr. 2009.

[17] T. Isernia, F. J. Ares Pena, O. M. Bucci, M. D’Urso, J. F. Gomez, and J. A. Rodriguez, "A hybrid approach for the optimal synthesis of pencil beams through array antennas," IEEE Trans. Antennas Propag., vol. 52, no. 11, pp. 2912-2918, Nov. 2004.

[18] Ching-Yih Tseng, Member, IEEE, and Lloyd J. Griffiths, "A Unified Approach to the Design of Linear Constraints in Minimum Variance Adaptive Beamformers".

[19] B.P. Ng M.H. Er C. Kot "Linear array geometry synthesis with minimum sidelobe level and null control".

[20] Shiann-Jeng Yu and Ju-Hong Lee, "Adaptive Array Beamforming Based on an Efficient Technique"

[21] Renbiao Wu, Zheng Bao and Yuanliang Ma "Control of Peak Sidelobe Level in Adaptive Arrays".

[22] Sotirios K. Goudos, George S. Miaris, Katherine Siakavara, and John N. Sahalos," On the Orthogonal Nonuniform Synthesis From a Set of Uniform Linear Array".

[23] Suhyun Park, Andrei B. Karpiouk, Salavat R. Aglyamov, Stanislav Y. Emelianov," Adaptive beamforming for photoacoustic imaging using linear arraytransducer".

[24] R. H. MacPhie, "Thinned coincident arrays for the direct measurement of the principal solution in radio astronomy," IEEE Trans. Antennas Propag., vol. AP51, pp. 788-793, Apr. 2003.

[25] "Transformations for nonideal uniform circular arrays operating in correlated signal environments," IEEE Trans. Signal Processing, vol. 54, no. 1, pp. 34-48, Jan. 2006. 